\title{
Complejidad sintáctica y textual en niños con trastorno por déficitt de atención/hiperactividad
}

\author{
Beatriz Gallardo-Paúls, Verónica Moreno-Campos, Patricia Roca, José L. Pérez-Mantero
}

Introducción y objetivos. En este trabajo se analiza la sintaxis utilizada por niños con diagnóstico de trastorno por déficit de atención/hiperactividad (TDAH) en la redacción de textos de superestructura narrativa y argumentativa. Los datos básicos analizados son la ratio de complejidad sintáctica y la distribución relativa de los tiempos verbales, asumiendo que en las lenguas fusionantes como el español el sistema TAM (tiempo, aspecto, modo) es el correlato lingüístico directo de las funciones ejecutivas relacionadas con la gestión de la temporalidad.

Sujetos y métodos. Se solicitó a niños de entre 8 y 13 años la elaboración de textos narrativos y argumentativos escritos; los datos se obtuvieron en contextos clínicos. Se analizó cualitativa y cuantitativamente la utilización de estructuras sintácticas complejas y de la conjugación verbal.

Resultados y conclusiones. Los niños con TDAH desarrollan valores menores en los niveles de complejidad sintáctica y utilizan menos subordinadas que los niños del grupo control en ambos tipos de texto; por lo que se refiere a los tiempos verbales, emplean en mayor proporción los tiempos prototípicos, es decir, presente de indicativo en la argumentación e imperfecto de indicativo en la narración.

Palabras clave. Déficit sintáctico. Pragmática textual. TDAH.

\section{Introducción y objetivos}

La descripción del uso lingüístico en hablantes con diagnóstico de trastorno por déficit de atención/hiperactividad (TDAH) ha señalado un déficit que afecta especialmente a los componentes sintáctico y pragmático [1-4]. A partir de la base de datos de hablantes infantiles y adolescentes con TDAH pertenecientes al corpus PerLA de lenguaje deficitario [5], nuestros estudios previos han atendido especialmente a elementos propios de pragmática textual. Así, a partir de datos escritos de naturaleza argumentativa y narrativa, hemos comprobado [6] que en las edades iniciales de la competencia escrita (grupo entre 7 y 11 años) los hablantes con TDAH presentan déficits pragmáticos en los tres niveles de organización informativa: los sucesos y episodios (se omiten algunos elementos y otros se vinculan de manera incoherente), las descripciones (en general, apenas se proporciona información de anclaje contextual) y las evaluaciones (también predominan las ausencias); igualmente, se registran vacilaciones importantes en el uso de los conectores pragmáticos que vinculan las distintas categorías del nivel de sucesos. En esta misma franja de edad [7], el estudio de las huellas formales de la capacidad intersubjetiva (o teoría de la mente) nos muestra que los niños con TDAH utilizan menos estas marcas que los referentes del grupo control, tanto en los formatos narrativos como argumentativos; pero la diferencia es cuantitativa, no cualitativa, y afecta a las cuatro manifestaciones básicas de la capacidad intersubjetiva: el léxico específicamente mentalista, las argumentaciones mentalistas implícitas, la emergencia textual de los sujetos de la enunciación y la polifonía textual. Al comparar específicamente los usos argumentativos infantiles con los adolescentes [8], se comprueban las mismas distancias entre el grupo con TDAH y el grupo control (en ambas franjas de edad el grupo con TDAH utiliza doblemente las estrategias de sanción, especialmente negativa), pero encontramos diferencias entre ambas fases: por un lado, en la adolescencia desaparece el predominio de argumentaciones de sanción (premio o castigo) de la edad infantil, y aumentan los usos de argumentación lógica y las falacias circulares; por otro lado, en la edad infantil los hablantes con TDAH emplean menos argumentaciones que los del grupo control, mientras que en edades adolescentes hallamos que los hablantes con TDAH aducen un mayor número de justificaciones para cada tesis.

A partir de este análisis eminentemente pragmático, en este trabajo se plantea cuál es la dimensión sintáctica de estas alteraciones pragmáticas en niños y adolescentes con TDAH, ya que las estruc-
Universitat de València (B. GallardoPaúls, J.L. Pérez-Mantero). Universitat Internacional Valenciana (V. Moreno-Campos). Instituto Valenciano de Neurología Pediátrica, INVANEP (P. Roca) Valencia, España.

\section{Correspondencia:}

Dra. Beatriz Gallardo Paúls. Facultad de Filología, Traducción y Comunicación. Universitat de

València. Avda. Blasco Ibáñez, 32, 5.‥ E-46010 Valencia.

Fax:

+34963864778.

E-mail:

beatriz.gallardo@uv.es

Financiación:

Trabajo realizado en el marco de los proyectos 'Coherencia, cohesión y pragmática textual en situaciones de déficit lingüístico' (MICINN FFI2008-02592/FILO) y

'Capacidades metalingüísticas y función ejecutiva en afasias y trastornos relacionados. Estudio interdisciplinar y evaluación clínica' (Ref. FFI2011-25947)

Declaración de intereses: Los autores manifiestan la inexistencia de conflictos de interés en relación con este artículo.

\section{Aceptado tras revisión externa:} 31.01.12.

Cómo citar este artículo: Gallardo-Paúls B, Moreno-Campos V, Roca P, Pérez-Mantero JL. Complejidad sintáctica y textual en niños con trastorno por déficit de atención/hiperactividad. Rev Neurol 2012; 54 (Supl 1): S131-5.

(c) 2012 Revista de Neurología 
turas sintácticas son el reflejo formal inmediato del procesamiento cognitivo. En concreto, intentamos profundizar en la descripción del déficit sintáctico en estos hablantes, con especial atención a la complejidad sintáctica de los enunciados y al uso de los tiempos verbales, asumiendo los planteamientos ya clásicos de Barkley que establecen la estrecha relación entre la sintaxis, la gestión de la temporalidad y memoria de trabajo y la corteza prefrontal. Barkley sigue la propuesta de Fuster sobre la corteza prefrontal como encargada de la organización temporal (cross-temporal) de la conducta, y señala que, mientras que los niños sólo pueden anticiparse a hechos futuros cercanos en el tiempo, los adolescentes y adultos pueden prepararse para acontecimientos más alejados del tiempo de la enunciación. Así pues, la sintaxis refleja la organización temporal del hablante y su gestión de los procesos cognitivos de anticipación y retrospectiva. Como indican Lambalgen et al [9], las incoherencias discursivas de hablantes con TDAH, manifestadas especialmente en el uso sintáctico de los tiempos verbales, se pueden explicar desde los planteamientos que asumen que el TDAH es una alteración de la función ejecutiva (concepto tan mal definido como el propio TDAH, según dicen) que implicaría problemas con la planificación.

Desde este punto de vista, la gestión verbal de la temporalidad asociada a los tiempos verbales de la gramática puede considerarse un rasgo formal relevante en el lenguaje de hablantes con TDAH. Por decirlo en palabras de Barkley: 'The present elicits a past from which is conjectured a future, the anticipation of which controls our behavior.

\section{Sujetos y métodos}

Del conjunto de datos escritos por hablantes con TDAH que integran el corpus PerLA, se han seleccionado para este estudio, con criterios de edad, 41 textos argumentativos y 46 textos narrativos tanto de hablantes con TDAH como del grupo control, con edades entre 8 y 13 años. Los datos proceden de diversos centros clínicos con los que colabora el grupo de investigación, entre los que destaca el Instituto Valenciano de Neurología Pediátrica (INVA$\mathrm{NEP}$ ), por proporcionar la mayor parte de la muestra; para los datos de control se contactó con dos colegios públicos de la ciudad de Valencia.

Como se ha explicado en otros lugares, se utilizan ejemplos de composiciones narrativas y argumentativas a partir de la creencia de que ambos tipos de superestructura reflejan distintos modos de procesamiento cognitivo $[10,11]$. Además, la dife- rente naturaleza de cada tipo de texto favorece una extensión mayor o menor y la utilización predominante de determinados tiempos y modos verbales.

La obtención de datos narrativos se realizó a partir de un taller ('La fábrica de cuentos') en el que se proporcionaba al informante un número cerrado de categorías narrativas (héroe, punto de partida, objetivo, amigo, objeto mágico, enemigo y lugar) a partir de las cuales debía escribir una historia; para los datos argumentativos se diseñaron tareas concretas que pedían a los informantes el desarrollo de textos argumentados a favor o en contra de cierta postura (comentar quién tiene una conducta incorrecta en cierta imagen, argumentar a favor o en contra de propuestas relativas a normas de conducta en el centro escolar, videojuegos preferidos...).

Para los informantes procedentes de INVANEP, se ha documentado información específica de carácter neuropsicológico con la intención de realizar ulteriores estudios que completen el presente trabajo [12]. Esta información incluye:

- Existencia o no de medicación.

- Criterios de impulsividad, hiperactividad o inatención que cumple según el Manual diagnóstico y estadístico de los trastornos mentales, cuarta edición (a partir de la información proporcionada por sus padres o maestros).

- Cociente intelectual total medido con la Wechsler Intelligence Scale for Children Revised.

- Respuestas correctas y errores en el Continuous Performance Test II (errores de omisión inesperada, de comisión simple, de comisión no-target y de espera).

- Datos del test de Stroop en la modalidad palabra/color (errores congruentes e incongruentes).

- Tiempo total y errores cometidos en el Matching Familiar Figures Test 20 (errores globales, errores de impulsividad y de ineficiencia).

- Resultado total de la subprueba de comprensión de sintaxis de la batería de lenguaje objetiva y criterial (puntuación directa).

Por lo que se refiere a la medicación, todas las pruebas neuropsicológicas se llevaron a cabo en la fase previa al tratamiento; en cambio, las pruebas de redacción lingüística se han efectuado siempre en torno a los seis meses de tratamiento, y las valoraciones suelen realizarse a última hora de la tarde.

\section{Resultados}

Para cada texto, se han analizado aspectos relacionados con su extensión (número de palabras y nú- 
mero de oraciones por texto) y la utilización de los distintos tiempos verbales. Pero además de estos valores absolutos se ha considerado también la complejidad sintáctica de los textos en cuestión, es decir, la ratio de subordinación empleada en los textos, así como los niveles de incrustación. También se han analizado las diferencias cuantitativas de los usos de los dos grupos en todas las respuestas con el programa SPSS v. 19.

Por lo que se refiere a la longitud estricta de los textos, los de hablantes con TDAH presentan una longitud media de 41,9 palabras en las argumentaciones escritas planificadas (AEP) y de 85,4 palabras en las narraciones escritas planificadas (NEP), que se oponen a un promedio de 95 palabras por texto en las AEP y de 162,3 palabras por texto en las NEP de los hablantes del grupo control. Esta diferencia tan notable en la longitud de los textos podría verse parcialmente justificada por las condiciones de obtención de éstos, ya que se realizaban en el entorno escolar con los niños del grupo control y en el entorno de la clínica con los niños con TDAH, y, por tanto, con menos tiempo; pero es ya un rasgo que indica menos efectividad en el desarrollo de la tarea propuesta, y que aparece también en estudios similares, donde la extensión de los textos del grupo control casi duplica la de los textos del grupo con TDAH [11]. Ambos grupos, no obstante, muestran más fluidez en la construcción de narraciones, lo que evidencia la mayor familiaridad y comodidad con esta superestructura textual (algo que es general a todos los informantes de todas las edades, incluida la muestra de adolescentes que llega hasta los 17 años). Al revisar estos valores en términos sintácticos, considerando por tanto el número de oraciones en que cada autor organiza su texto, se observa que las argumentaciones y narraciones de los niños con TDAH tienen un promedio ligeramente mayor de oraciones por texto: 3,5 por AEP y 6,2 oraciones por NEP, frente a las 3,3 por AEP y 5,5 oraciones por NEP en los textos del grupo control; no obstante, éste no es un dato significativo.

Tanto en los textos narrativos (media: 123,80) como argumentativos (media: 68,40) se encuentra una diferencia importante a favor del grupo control $(U=1,501 ; z=6,126 ; p=0,000$ y $U=1,476 ; z=$ 5,$895 ; p=0,000$; respectivamente) en lo que puede denominarse el nivel de complejidad sintáctica, con lo que se hace referencia a la ratio entre oraciones principal y subordinadas. En los datos del grupo con TDAH existe menos uso de la subordinación, especialmente en los textos narrativos (en ambos grupos se dan valores inferiores al uso de una subordinada por oración), mientras que la argumen-
Figura 1. Usos predominantes de tiempos verbales en textos argumentativos.

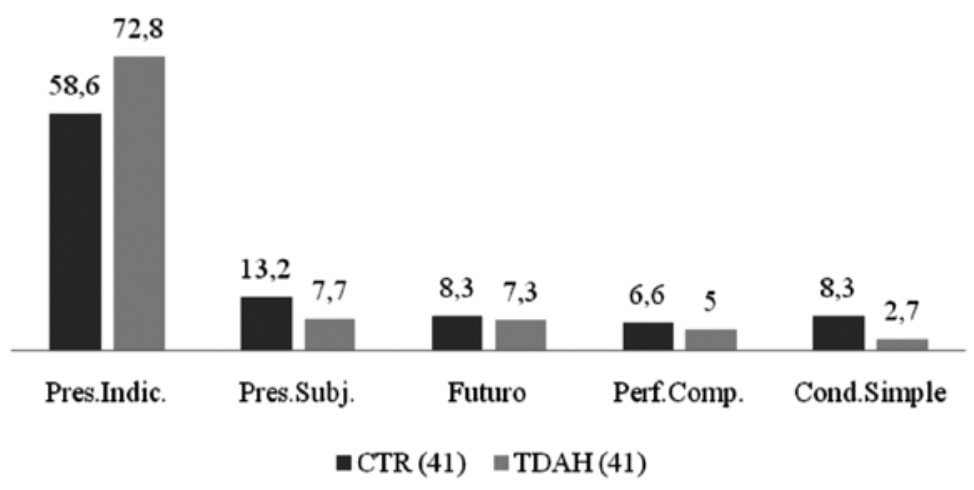

tación privilegia el uso de subordinadas causales desde el mismo planteamiento de la tarea, y, por tanto, presenta una ratio superior (en ambos grupos: valores $>1$ ).

Los usos verbales presentan repartos diferentes según los tipos de texto, y esto se manifiesta en los dos grupos de informantes, donde hemos señalado los resultados que han sido significativos. Para los textos argumentativos, en la figura 1 se halla la distribución de los tiempos verbales. En el presente de indicativo resulta significativo el mayor uso en el grupo con TDAH (media: 41,27; $U=461 ; z=-3,521$; $p=0,000)$. En el presente de subjuntivo, el uso por parte del grupo control (media: 6,05) resulta significativo $(U=1,138 ; z=3,068 ; p=0,002)$, así como en el condicional simple (media: 3,$10 ; U=1,103 ; z=$ 3,073; $p=0,002)$.

En los textos narrativos, la distribución se refleja en la figura 2; en el condicional simple, el mayor uso del grupo control (media: 0,74) resulta significativo $(U=1,037 ; z=2,787 ; p=0,005)$.

Como puede apreciarse en las figuras, los textos argumentativos privilegian el uso de formas de presente, que es el tiempo verbal prototípico en esta superestructura; en los textos del grupo con TDAH se evidencia un mayor uso de esta forma en detrimento de tiempos verbales más específicos que sí se rentabilizan en el grupo control. Por lo que se refiere a las narraciones, existe equilibrio en ambos grupos para el uso predominante del pretérito perfecto simple, y el uso compensatorio por parte del grupo con TDAH recae en el pretérito imperfecto, que es la segunda forma prototípica. Las diferencias entre ambos tipos de texto tienen una evidente explicación en términos de lingüística cognitiva y teo- 
Figura 2. Usos predominantes de tiempos verbales en textos narrativos.

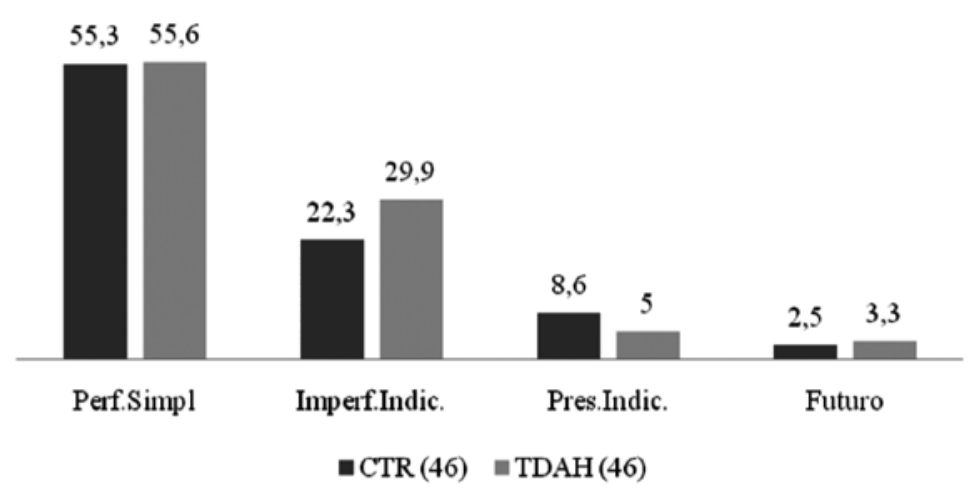

ría de prototipicidad. Es evidente que las distintas superestructuras textuales tienen una orientación cognitiva específica que cristaliza en el predominio de unos u otros tiempos verbales, pues la argumentación se orienta al marco intemporal del presente, en la medida en que las opiniones y las ideas que la alimentan se vinculan al tiempo de la enunciación; de ahí el predominio del presente de indicativo en los textos del grupo control, que se confirma en los textos del grupo con TDAH. Por su parte, la narración es prototípicamente un tipo textual orientado a tiempos pasados $y$, preferiblemente, de aspecto perfectivo, como el pretérito perfecto simple, que predomina en ambos grupos. Como puede apreciarse, tanto el presente de indicativo como el pretérito perfecto simple son, además, tiempos verbales absolutos, no condicionados por el uso de otras formas del mismo texto (sólo dependen del tiempo de la enunciación).

\section{Discusión y conclusiones}

El análisis sintáctico de textos escritos por niños con diagnóstico de TDAH revela que su utilización de la subordinación es ligeramente menor que en sus pares de edad de desarrollo típico, si bien, como se apreciaba también en los análisis de categorías pragmáticas, las diferencias en las edades estudiadas son básicamente cuantitativas, y no cualitativas. Por lo que se refiere a la distribución del uso de los tiempos verbales, se aprecia también que los niños con TDAH muestran un reparto menos elaborado, de manera que se apoyan más en los tiempos prototípicos para ambas superestructuras (presente de indicativo para la argumentación y pretérito imperfecto para la narración).

En definitiva, el análisis de la sintaxis en el TDAH permite completar estudios previos de naturaleza pragmática mediante la valoración de los niveles de complejidad sintáctica (ratio de subordinación) y la distribución relativa de los tiempos verbales utilizados. La relevancia de este tipo de estudio se vincula directamente con los planteamientos que, a partir de Barkley, enfatizan los problemas de gestión de la temporalidad en el TDAH, pues tanto los usos temporales como la secuencialidad ordenada de éstos pueden considerarse un correlato lingüístico de las funciones ejecutivas que gestionan la temporalidad y la organización subjetiva.

Bibliografía

1. Baker L, Cantwell D. Attention deficit disorder and speech/ language disorders. Comprehensive Mental Health Care 1992; 2: 3-16.

2. Barkley RA. Impaired delayed responding: a unified theory of attention deficit hyperactivity disorder. In Routh DK, ed. Disruptive behavior disorders in childhood: essays honoring Herbert C. Quay. New York: Plenum; 1994. p. 11-57.

3. Barkley RA. ADHD and the nature of self-control. New York: Guilford Press; 1997.

4. Tirosh E, Cohen A. Language deficit with attention-deficit disorder: a prevalent comorbidity. J Child Neurol 1998; 13: 493-7.

5. Gallardo-Paúls B. Trastorno por déficit de atención y/o hiperactividad. Materiales y análisis pragmático. Valencia: Universitat de València; 2009.

6. Gallardo-Paúls B. Niveles de organización narrativa en TDAH. IX Curso Internacional de Actualización en Neuropediatría y Neuropsicología infantil. Valencia, España, 1-3 de marzo de 2007.

7. Gallardo-Paúls B. Las huellas lingüísticas de teoría de la mente: intersubjetividad y enunciación. Rev Neurol 2008; 46 (Supl 1): S29-35.

8. Gallardo-Paúls B, Moreno-Campos V, Gimeno-Martínez M. Pragmática textual en adolescentes con trastorno por déficit de atención/hiperactividad: argumentación. Rev Neurol 2010; 50 (Supl 3): S113-7.

9. Lambalgen M, Kruistum K, Parigger E. Discourse processing in attention-deficit hyperactivity disorder (ADHD). J Log Lang Inf 2008; 17: 467-87.

10. Bruner JS. Explaining and interpreting: two ways of using mind. In Harman G, ed. Conceptions of the human mind: essays in honor of George A. Miller. Hillsdale: Lawrence Erlbaum; 1993. p. 123-36.

11. Mathers M. Some evidence for distinctive language use by children with attention deficit hyperactivity disorder. Clin Linguist Phon 2005; 19: 215-25.

12. Mulas F, Etchepareborda MC, Abad-Mas L, Díaz-Lucero AH, Hernández S, De la Osa-Langreo A, et al. Trastornos neuropsicológicos de los adolescentes afectos de trastorno por déficit de atención con hiperactividad. Rev Neurol 2006; 43 (Supl 1): S71-81.

13. Fernández-Pérez M. Child speech and language acquisition. Some topics revisited. Folia Linguistica 2012. [In press]. 


\section{Syntactic and textual complexity in children with attention deficit hyperactivity disorder}

Introduction and aims. In this work we analyse the syntax used by children diagnosed with attention deficit hyperactivity disorder (ADHD) when writing texts with a narrative and argumentative superstructure. The basic data analysed are the ratio of syntactic complexity and the relative distribution of verb tenses, assuming that in fusional languages like Spanish the TAM system (tense, aspect, mode) is the direct linguistic correlate of the executive functions related with the management of temporality.

Subjects and methods. Children between 8 and 13 years of age were asked to write narrative and argumentative texts; data were obtained in clinical contexts. The use of complex syntactic structures and verb conjugation were analysed both quantitatively and qualitatively.

Results and conclusions. The children with ADHD displayed lower values in terms of levels of syntactic complexity and used fewer subordinate clauses than the children in the control group in both types of texts. As far as verb tenses are concerned, they used a higher proportion of prototypical tenses, that is to say, the present simple in argumentation and the imperfect indicative in narrative texts.

Key words. ADHD. Syntactic deficit. Textual pragmatics. 